\title{
First-Principles Study of Magnetic Properties of Co/Pt(111) Film in Electric Field
}

\author{
Sho YAsuda and Shugo SuzukI \\ Division of Materials Science, Faculty of Pure and Applied Sciences, University of Tsukuba, \\ Tsukuba, Ibaraki 305-8573, Japan
}

KEYWORDS: Co/Pt(111), magnetic properties, electric field, first-principles calculations

Technology of electric field (EF) control of magnetic properties is attractive for spintronic devices. In particular, it has been shown that the EF can change the magnetization and the magnetic anisotropy. ${ }^{1-3)}$ According to an experimental study, ${ }^{2)}$ an EF less than $0.1 \mathrm{~V} / \mathrm{nm}$ causes a large change of $40 \%$ in the magnetic anisotropy energy (MAE) of the $\mathrm{Fe} / \mathrm{Au}(001)$ film. Also, another experimental study ${ }^{3)}$ using the $\mathrm{Co} / \mathrm{Pt}(111)$ film in the $\mathrm{EF}$ has shown that the Curie temperature of the film is changed up to $6 \mathrm{~K}$ by applying an EF of about $+0.2 \mathrm{~V} / \mathrm{nm}$ while down to $-6 \mathrm{~K}$ by applying an EF of about $-0.2 \mathrm{~V} / \mathrm{nm}$.

A first-principles study of the MAE in the EF for the $\mathrm{Fe} / \mathrm{Pt}(001)$ film was carried out; ${ }^{4)}$ it has been found that the change in the MAE is in proportion to the EF with the slope rate of $0.03 \mathrm{meV} / \mathrm{Fe}$ per $\mathrm{V} / \mathrm{nm}$ for $\mathrm{Fe} / \mathrm{Pt}(001)$. Another first-principles study of the MAE in the $\mathrm{EF}$ for the Fe monolayer was carried out; ${ }^{5}$ it has been found that the MAE is affected considerably by applying the EF. On the other hand, to our knowledge, there are no first-principles studies of the $\mathrm{Co} / \mathrm{Pt}(111)$ film in the $\mathrm{EF}$ although the Co films are as important as the Fe films.

In this study, we investigate the magnetic properties of the $\mathrm{Co} / \mathrm{Pt}(111)$ film in the EF carrying out the first-principles calculations. We take account of the EF by introducing electrode far above $\mathrm{Co} / \mathrm{Pt}(111)$ and changing the total number of electrons in the film. We calculate the spin moments and the numbers of electrons of the constituent atoms as well as the MAE of the film self consistently.

We employ the fully relativistic full-potential linear-combination-of-atomic-orbitals method based on the density-functional theory within the local spin density approximation. ${ }^{6}$ The exchange-correlation energy functional used in this study is the Perdew-Wang parameterization of the Ceperley-Alder results. ${ }^{7,8)}$

As shown in Fig.1, the Pt(111) surface is modeled by a 3-layer slab. We denote the Pt atoms in the top, middle, and bottom Pt layers by $\mathrm{Pt}(\mathrm{I}), \mathrm{Pt}(\mathrm{II})$, and $\mathrm{Pt}(\mathrm{III})$, respectively. We 
assume hcp stacking of the Co adlayer on the Pt(111) surface because the Co adatom prefers to occupy an hcp-hollow site on the Pt(111) surface. ${ }^{9)}$ We use the lattice constant of the triangular lattice, $2.78 \AA$, which is calculated from the lattice constant of fcc Pt, $3.92 \AA$. All the interlayer distances between adjacent atomic layers of $\mathrm{Co} / \mathrm{Pt}(111)$ are optimized calculating the forces acting on the Co and Pt atoms in the absence of the EF. The optimized interlayer distances are shown in Fig.1.

The EF is applied as follows. We change the total number of electrons in the film; we denote this change per Co atom by $\Delta N$. We introduce the following external potential originated in the electrode shown in Fig.1:

$$
V_{\mathrm{EF}}(z)=-\frac{2 \pi e^{2} \Delta N}{A_{\text {cell }}} z
$$

Here, $A_{\text {cell }}$ is the area of the unit cell of the triangular lattice. We also use the two-dimensional Ewald method. ${ }^{10,11)}$ All the atomic positions are fixed at those optimized in the absence of the EF.

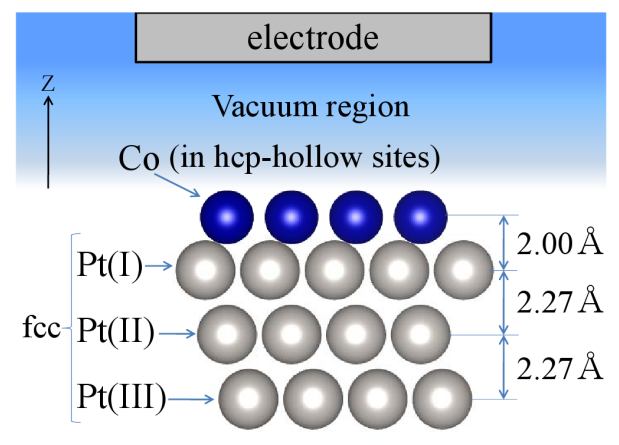

Fig. 1. (Color online) Schematic diagram of the computational model. The model constitutes of the $\mathrm{Co} / \mathrm{Pt}(111)$ film and the electrode. The Co monolayer is on the $\mathrm{Pt}(111)$ substrate. The Co atoms are in the hcp-hollow sites and the $\mathrm{Pt}(111)$ substrate is composed of three Pt layers.

We evaluate the MAE from the difference between the total energies for the easy and hard axis magnetization directions: $\mathrm{MAE}=E_{\mathrm{tot}}^{[110]}-E_{\mathrm{tot}}^{[111]}$, where the easy and hard axes are the [111] and [1ํㅣㄹ directions, respectively. The dipolar contribution to the MAE is not considered. The basis functions adopted in this study consist of the following atomic orbitals: $1 \mathrm{~s}, 2 \mathrm{~s}, 2 \mathrm{p}, 3 \mathrm{~s}, 3 \mathrm{p}, 3 \mathrm{~d}$, and $4 \mathrm{~s}$ orbitals of the neutral Co atom, 3d, 4s, and $4 \mathrm{p}$ orbitals of the $\mathrm{Co}^{2+}$ atom, $1 \mathrm{~s}, 2 \mathrm{~s}, 2 \mathrm{p}, 3 \mathrm{~s}, 3 \mathrm{p}, 3 \mathrm{~d}, 4 \mathrm{~s}, 4 \mathrm{p}, 4 \mathrm{~d}, 4 \mathrm{f}, 5 \mathrm{~s}, 5 \mathrm{p}, 5 \mathrm{~d}$, and $6 \mathrm{~s}$ orbitals of the neutral $\mathrm{Pt}$ atom, and $5 \mathrm{~d}, 6 \mathrm{~s}$, and $6 \mathrm{p}$ orbitals of the $\mathrm{Pt}^{2+}$ atom. The Brillouin-zone integration is carried out using the good-lattice-point method with $610 \boldsymbol{k}$ points. The convergence of the MAE with 
respect to the number of $\boldsymbol{k}$ points is about $0.04 \mathrm{meV} / \mathrm{Co}$, which occurs almost systematically with the same set of $\boldsymbol{k}$ points, irrespective of the magnitude of the EF; hence, this error does not affect the dependence of the MAE on the EF very much. To speed up the convergence, we use a modest Fermi distribution smearing of eigenstates with a small width of $30 \mathrm{meV}$.

The calculated spin moments of the $\mathrm{Co}, \mathrm{Pt}(\mathrm{I}), \mathrm{Pt}(\mathrm{II})$, and $\mathrm{Pt}(\mathrm{III})$ atoms are shown in Fig.2(a), (b), (c), and (d), respectively. Also, the calculated spin moments of the Co $3 \mathrm{~d}$ and Co 4s electrons are shown in Fig.3(a) and (b), respectively. The spin moments of the Co and $\mathrm{Pt}(\mathrm{I})$ atoms decrease with increasing $\Delta N$. On the contrary, the spin moments of the $\mathrm{Pt}(\mathrm{II})$ and $\mathrm{Pt}(\mathrm{III})$ atoms increase with increasing $\Delta N$. Also, the spin moments of the Co $3 \mathrm{~d}$ and Co $4 \mathrm{~s}$ electrons decrease with increasing $\Delta N$. The sum of the spin moments of the Co $3 \mathrm{~d}$ and Co $4 \mathrm{~s}$ electrons is almost equal to the spin moment of the Co atom for each $\Delta N$.

(a)

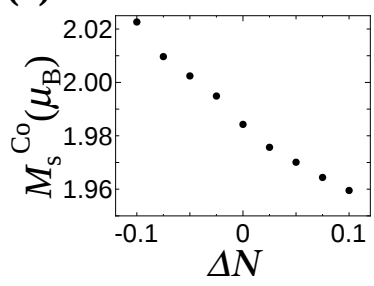

(c)

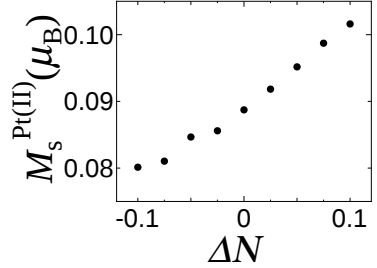

(b)

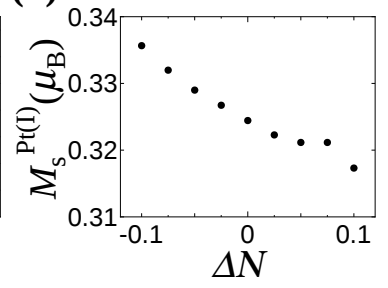

(d)

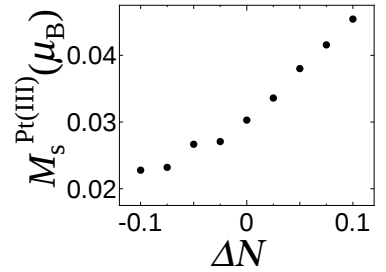

Fig. 2. Spin moments of (a) $\mathrm{Co}$, (b) $\mathrm{Pt}(\mathrm{I})$, (c) $\mathrm{Pt}(\mathrm{II})$, and (d) $\mathrm{Pt}(\mathrm{III})$ atoms as a function of external electric field.

(a)

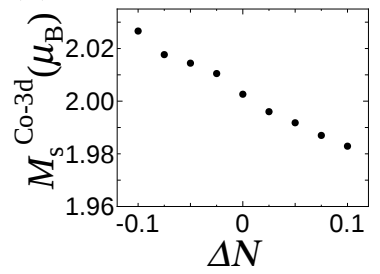

(b)

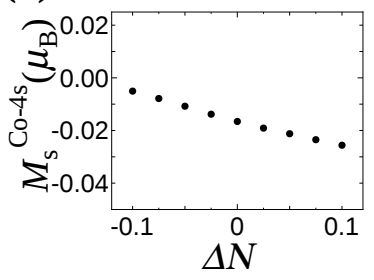

Fig. 3. Spin moments of (a) Co $3 d$ and (b) Co 4 s electrons as a function of external electric field.

The calculated numbers of electrons of the $\mathrm{Co}, \mathrm{Pt}(\mathrm{I}), \mathrm{Pt}(\mathrm{II})$, and $\mathrm{Pt}(\mathrm{III})$ atoms are shown 
Fig.4(a), (b), (c), and (d), respectively. Also, the calculated numbers of electrons of the Co 3d and $\mathrm{Co} 4 \mathrm{~s}$ electrons are shown in Fig.5(a) and (b), respectively. The number of electrons of the Co atom increases with increasing $\Delta N$. The number of electrons of the $\mathrm{Pt}(\mathrm{I})$ atom depends very little on $\Delta N$. On the other hand, the number of electrons of the $\mathrm{Pt}(\mathrm{II})$ atom increase with increasing $\Delta N$ while the number of electrons of the $\mathrm{Pt}(\mathrm{III})$ atom decrease with increasing $\Delta N$. Also, the number of the Co $3 \mathrm{~d}$ electrons is almost unchanged; this does not contradict the decrease in their spin moment, $-0.04 \mu_{\mathrm{B}}$, which is the difference between $\Delta N=-0.1$ and 0.1 shown in Fig.3(a), because one half of this decrease is due to a slight increase in the number of the Co $3 \mathrm{~d}$ electrons shown in Fig.5(a), +0.02, and the other half is due to their redistribution. On the other hand, the number of the Co $4 \mathrm{~s}$ electrons is changed noticeably; this change explains the change in the number of electrons of the Co atom shown in Fig.4.(a). The reason for this behavior might be that, compared with the Co $3 \mathrm{~d}$ electrons, the Co $4 \mathrm{~s}$ electrons play a major role in the screening of the EF.

(a)

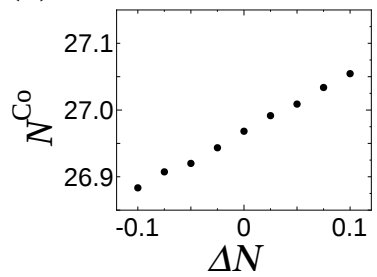

(c)

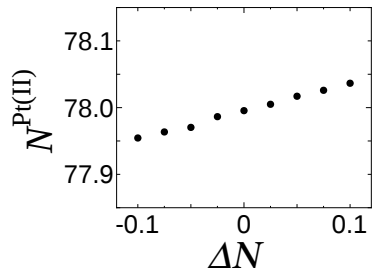

(b)

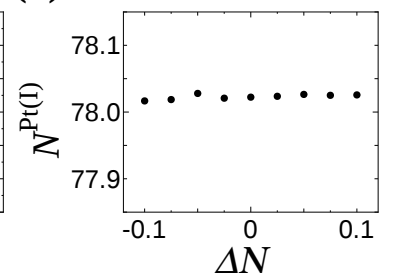

(d)

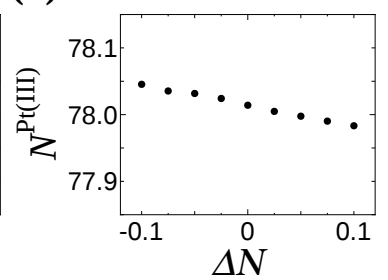

Fig. 4. Numbers of electrons of (a) Co, (b) $\mathrm{Pt}(\mathrm{I})$, (c) $\mathrm{Pt}(\mathrm{II})$, and (d) $\mathrm{Pt}(\mathrm{III})$ atoms as a function of external electric field.

We now discuss the dependence of the MAE on the EF. The calculated MAE depend very little on the EF within the range of $\Delta N$ considered in this study; the calculated MAE is $0.76 \pm 0.03 \mathrm{meV} / \mathrm{Co}$. Fitting a straight line to the calculated MAE as a function of the EF, the slope rate is found to be $-0.0004 \pm 0.0008 \mathrm{meV} / \mathrm{Co}$ per $\mathrm{V} / \mathrm{nm}$. The experimental study of $\mathrm{Fe} / \mathrm{Au}(001)$ indicates that the slope rate is about $0.02 \mathrm{meV} / \mathrm{Fe}$ per $\mathrm{V} / \mathrm{nm} .^{2)}$ The large change in the Curie temperature due to the EF observed in the experimental study of $\mathrm{Co} / \mathrm{Pt}(111)$ also indicates a large change in the MAE. ${ }^{3)}$ The theoretical study of $\mathrm{Fe} / \mathrm{Pt}(001)$ has shown that 
(a)

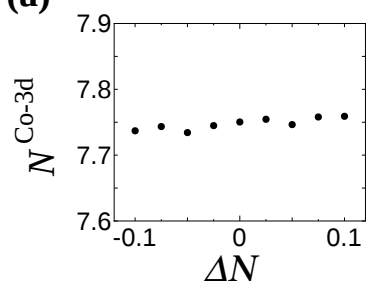

(b)

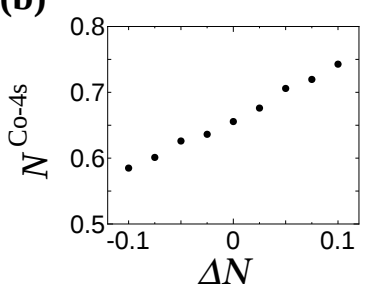

Fig. 5. Numbers of (a) Co $3 \mathrm{~d}$ and (b) Co 4 s electrons as a function of external electric field.

the slope rate is $0.03 \mathrm{meV} / \mathrm{Fe}$ per $\mathrm{V} / \mathrm{nm}^{4}{ }^{4)}$ Compared with these results, our calculated MAE show almost no dependence on the EF.

This might be originated in the difference between $\mathrm{Fe}$ and Co. Another possibility is that we did not take account of the structure change of $\mathrm{Co} / \mathrm{Pt}(111)$ due to the application of the $\mathrm{EF}$ or the insulator layer on $\mathrm{Co} / \mathrm{Pt}(111)$. In particular, the structure change due to the $\mathrm{EF}$ can alter the dependence of the MAE on the EF. We need to study the effects of the EF on the MAE, taking these points into account in the future. 


\section{References}

1) M. Weisheit, S. Fähler, A. Marty, Y. Souche, C. Poinsignon, and D. Givord: Science 315 (2007) 349.

2) T. Maruyama, Y. Shiota, T. Nozaki, K. Ohta, N. Toda, M. Mizuguchi, A. A. Tulapurkar, T. Shinjo, M. Shiraishi, S. Mizukami, Y. Ando, and Y. Suzuki: Nature Nanotech. 4 (2009) 158.

3) D. Chiba, S. Fukami, K. Shimamura, N. Ishiwata, K. Kobayashi, and T. Ono: Nature Mater. 10 (2011) 853.

4) M. Tsujikawa and T. Oda: Phys. Rev. Lett. 102 (2009) 247203.

5) K. Nakamura, R. Shimabukuro, Y. Fujiwara, T. Akiyama, T. Ito, and A. J. Freeman: Phys. Rev. Lett. 102 (2009) 187201.

6) S. Suzuki and K. Nakao: J. Phys. Soc. Jpn. 68 (1999) 1982.

7) J. P. Perdew and Y. Wang: Phys. Rev. B 45 (1992) 13244.

8) D. M. Ceperley and B. J. Alder: Phys. Rev. Lett. 45 (1980) 566.

9) P. Błoński and J. Hafner: J. Phys.: Condens. Matter 21 (2009) 426001.

10) D. E. Parry: Surf. Sci. 49 (1975) 433.

11) F. E. Harris: Int. J. Quantum Chem. 68 (1998) 385. 